\title{
PDP INSIGHTS
}

\section{The Predominant Maternal Contribution to the Inflammatory Response in VUE and Syphilitic Infectious Villitis}

Between 5 and 10\% of placentas feature a chronic inflammatory reaction involving the chorionic villi, whose etiology is, in most cases (approximately 95\%), undetermined (VUE). The remaining $5 \%$ of chronic villitis cases have an infectious cause. The origin of the infiltrating inflammatory cells in VUE has been previously established as maternal, raising several theories about its pathogenesis. However, no studies have addressed the question of fetal versus maternal origin of the inflammatory response in cases of infectious villitis. Kapur et al. have tackled this problem and present their results in the current issue (pages 453-458, DOI: 10.1007/s10024-004-2124-3). Using two cases of syphilitic infectious villitis, and four of VUE, all of which involved male fetuses, the authors employed a combination of immunohistochemistry for different leukocyte markers, and fluorescent in situ hybridization to detect $\mathrm{X}$ and $\mathrm{Y}$ chromosomes, to establish the type and origin of the infiltrating inflammatory cells. The results of this study contradict the long standing belief that inflammation of infectious etiology is of fetal origin. These authors found that in both, infectious and VUE cases, infiltrating cells were of material derivation; this also included neutrophils in foci of acute villitis. B-lymphocytes, although present, were scarce in both types of villitis. These findings change our views about the immunologic/inflammatory response in cases of infectious villitis, and raise the possibility of a common response pattern in both types of inflammatory processes. Whether the inflammatory response in cases of syphilitic villitis represents a direct maternal response against the treponema, an alloimmune reaction similar to VUE, potentiated by infection, or an infection-driven cytokine effect remains to be investigated. Although the number of cases studied is small, and further corroboration of these findings is desirable, the emerging evidence presented by these authors is convincing, and it sets the stage for additional studies, including examples of other types of infection-associated chronic villitis.

\section{Metapneumovirus, a Newly Described Respi- ratory Virus: First Description of Its Patho- logic Effects}

Human metapneumovirus (hMPV) was first described in 2001 as a member of the Paramyxoviridae family that causes respiratory infections in young children. Its clinical picture ranges from bronchiolitis of varying degrees to pneumonia, resembling that of Respiratory Syncytial Virus (RSV), a close relative of hMPV. So far, no descriptions of the cytologic, histologic, or ultrastructural feature associated with hMPV infection have been made. Therefore, the contribution by Vargas et al in the current issue (pages 478-486, DOI: 10.1007/s10024-004-1011-2) is a valuable addition to our understanding of this emerging respiratory pathogen. The study included 6 bronchioalveolar lavage specimens, and 3 lung biopsies obtained within four months of their hMPV detection assay by the Virology laboratory. At the cytological level, the changes included degeneration of the respiratory epithelial cells, necrosis, ciliacytophtoria (fragments of apical cytoplasm with attached cilia), and round cytoplasmic inclusions. There was a background of hemosiderophages, neutrophils, and mucus. Histologically, there was chronic airway inflammation and intraalveolar foamy histiocytes and hemosiderophages. Unfortunately, no viral particles were found in the specimens studied by electron microscopy. Based on their observations, the authors believe that hMPV infects primarily the airway epithelium and suggest a pathogenetic mechanism of direct infection of the airway lining cells leading to their degeneration and necrosis, followed by a neutrophilic response and an exaggerated mucus production. Later changes include a lymphoid tissue expansion, squamous metaplasia and other processes associated with a chronic healing response. These findings may overlap with those associated with other viruses infecting the respiratory tract, such as SRV, Parainfluenza virus, and measles. In addition, the peculiar fragmentation of ciliated cells may be confused with ameboflagellates, which may be found in nasal aspirates and sputum samples of allergic or asthmatic patients. The practicing pathologist will benefit from becoming familiar with this new form of pathogen and its associated pathology. 